\title{
REPRESENTATIVE VOLUME ELEMENT (RVE) BASED CRYSTAL PLASTICITY STUDY OF VOID GROWTH ON PHASE BOUNDARY IN TITANIUM ALLOYS
}

\author{
U. B. ASIM ${ }^{1 *}$, M. A. SIDDIQ ${ }^{1 *}$ and M. E. KARTAL ${ }^{1}$ \\ ${ }^{1}$ School of Engineering, University of Aberdeen, Fraser Noble Building, Aberdeen, AB24 3UE, UK \\ Emails: amir.siddiq@abdn.ac.uk \\ *Corresponding author
}

\begin{abstract}
Crystal plasticity based finite element method (CPFEM) studies have been successfully used to model different material behaviour and phenomenon, including but not limited to; fatigue, creep and texture evolution. This capability can be extended to include the ductile damage and failure in the model. Ductile failure in metals is governed by void nucleation, growth, and coalescence. High strength titanium alloys can be formed from sheets and components and are prone to ductile failure. $\alpha-\beta$ Titanium alloys are in widespread use, ranging from aerospace, automotive, energy to oil and gas. They have multiple phases present in the microstructure but $\alpha$ and $\beta$ phases are dominant and are present in various morphologies. This study focuses on the $3 D$ representative volume element ( $R V E$ ) simulations of spherical void of known initial porosity at the interface of $\alpha$ and $\beta$ phase single crystals. The effect of initial porosity, applied triaxiality and orientation of RVE with respect to the loading direction is investigated. Slip based crystal plasticity formulation implemented as a user subroutine in commercially available software was used to simulate the void growth and the results of the same are presented. Lastly, a generalised correlation among loading type, loading direction, crystal orientation, phase interface orientation, and void growth is presented.
\end{abstract}

Keywords: Crystal plasticity; phase boundary; void growth; titanium alloys; dual phase alloys

\section{INTRODUCTION}

Titanium alloys have been used in various application areas including, but not limited to, aerospace, automobile, biomedicine, process industry including oil and gas. $\alpha-\beta$ titanium alloys are the class of titanium alloys which have been widely used in the aerospace industry in larger quantities than other types because of their unique set of properties. Ductile fracture is an important consideration in their use and production [1-3]. It was established by the experiments carried out on Ti-10V-2Fe-3Al alloy which is an $\alpha-\beta$ titanium alloy, that void nucleation, growth and coalescence is the failure mechanism. Voids were found to nucleate on the phase boundaries between the $\alpha$ and $\beta$ phases and different morphology $\alpha$ phases [4-6].

This study comprises two aspects. In the first part unit cell calculations were carried out using fully validated crystal plasticity finite element method on RVEs having spherical void embedded at the interface of bicrystal of $\alpha$ and $\beta$ phase each. The effect of initial porosity, applied stress triaxiality and phase boundary inclination (PBI) (term will be explained later in the article) was studied and quantified. This method has been used to develop better understanding of the micromechanics and underlying phenomenon that leads to ductile failure in single crystals $[7,8,17,9-16]$; on the interface of polycrystals with different hardening rates [18]; on bicrystal of the same phase having face centred cubic (FCC) crystal structure [19] and body centred cubic (BCC) crystal structure [20], with different crystal orientations.

The second part of the presented work deals with the formulation of a model that predicts void growth in the form of evolution of normalised void volume fraction, for the voids present 
at the interface of dissimilar material. A model is proposed that depends on the material properties, initial porosity, applied strain, stress triaxiality, and PBI. A comparison between the unit cell results and the predictions of the proposed model has been presented.

\section{UNIT CELL CALCULATIONS}

\subsection{Crystal Plasticity Formulation}

The matrix material of the RVE around a void is modelled as a bicrystal, with properties of the single crystals of $\alpha$ and $\beta$ phases assigned to each half of the RVE. Single crystal behaviour is modelled using crystal plasticity formulation which incorporates anisotropic elastic and slip based deformation mechanisms. A brief overview of the formulation used is given here, details of which can be found elsewhere [21]. Deformation gradient is described as a product of elastic, $F^{e}$, and plastic, $F^{p}$, parts.

$$
\boldsymbol{F}=\boldsymbol{F}^{e} \boldsymbol{F}^{p}
$$

The elastic part can then be further decomposed into elastic stretch and rigid body rotation, resulting in:

$$
\boldsymbol{F}=\boldsymbol{V}^{e} \boldsymbol{R}^{e} \boldsymbol{F}^{p}
$$

The product of rigid body rotation and the plastic part is treated as an intermediate unloaded configuration, represented as $\boldsymbol{F}^{*}=\boldsymbol{R}^{e} \boldsymbol{F}^{p}$.

Velocity gradient, $\boldsymbol{l}$, is defined as:

$$
\boldsymbol{l}=\dot{\boldsymbol{F}} \boldsymbol{F}^{-1}
$$

Transformation to the intermediate configuration, $\tilde{\boldsymbol{L}}$, where $\widetilde{(\cdot)}$ represents the intermediate unloaded configuration, the total velocity gradient can be given by:

$$
\tilde{\boldsymbol{L}}=\boldsymbol{V}^{e^{-1}} \boldsymbol{l} \boldsymbol{V}^{e}
$$

Symmetric and skew-symmetric parts of $\widetilde{\boldsymbol{L}}, \widetilde{\boldsymbol{D}}$ and $\widetilde{\boldsymbol{W}}$ respectively, can then be defined as:

$$
\widetilde{\boldsymbol{D}}=\boldsymbol{V}^{e^{T}} \boldsymbol{d} \boldsymbol{V}^{e}, \widetilde{\boldsymbol{W}}=\boldsymbol{V}^{e^{T}} \boldsymbol{w} \boldsymbol{V}^{e}
$$

Where $\boldsymbol{d}$ and $\boldsymbol{w}$ are the symmetric and skew symmetric parts of total velocity gradient. These can then be additively decomposed into elastic and plastic contributions using:

$$
\widetilde{\boldsymbol{D}}=\dot{\widetilde{\boldsymbol{E}}}+\widetilde{\boldsymbol{D}}^{*}, \widetilde{\boldsymbol{W}}=\operatorname{skew}\left(\boldsymbol{V}^{e^{T}} \dot{\boldsymbol{V}}^{e}\right)+\widetilde{\boldsymbol{W}}^{*}
$$

Here $\widetilde{\boldsymbol{D}}^{*}$ and $\widetilde{\boldsymbol{W}}^{*}$ are the plastic contributions due to slip, skew $(\cdot)$ represents skew symmetric part of a quantity and $\dot{\widetilde{\boldsymbol{E}}}$ is the elastic strain rate tensor.

Anisotropic plasticity is used with the help of the second order tensor of elastic constants rotated to the unloaded configuration, $\tilde{\mathbb{C}}^{e}$, which relates 2 nd Piola-Kirchhoff stress tensor, $\tilde{\boldsymbol{S}}$, and applied elastic strain tensor, $\widetilde{\boldsymbol{E}}^{e}$, as:

$$
\tilde{\boldsymbol{S}}=\widetilde{\mathbb{C}}^{e} \widetilde{\boldsymbol{E}}^{e}, \widetilde{\mathbb{C}}^{e}=\left[\begin{array}{cccccc}
C_{11} & C_{12} & C_{13} & 0 & 0 & 0 \\
C_{12} & C_{11} & C_{13} & 0 & 0 & 0 \\
C_{13} & C_{13} & C_{33} & 0 & 0 & 0 \\
0 & 0 & 0 & C_{44} & 0 & 0 \\
0 & 0 & 0 & 0 & C_{44} & 0 \\
0 & 0 & 0 & 0 & 0 & C_{44}
\end{array}\right]
$$

Here $C_{i j}$ are the elastic coefficients for hexagonal close packed (HCP) single crystals. For BCC crystals, $C_{13}=C_{12}$ and $C_{33}=C_{11}$. Plastic response due to slip is defined as:

$$
\begin{aligned}
& \widetilde{\boldsymbol{D}}^{*}=\operatorname{sym}\left(\widetilde{\boldsymbol{C}}^{e} \widetilde{\boldsymbol{\Omega}}^{e}\right)+\sum_{\alpha=1}^{N} \dot{\gamma}^{\alpha} \operatorname{sym}\left(\widetilde{\boldsymbol{C}}^{e} \widetilde{\mathbf{Z}}^{\alpha}\right) \\
& \widetilde{\boldsymbol{W}}^{*}=\operatorname{skew}\left(\widetilde{\boldsymbol{C}}^{e} \widetilde{\boldsymbol{\Omega}}^{e}\right)+\sum_{\alpha=1}^{N} \dot{\gamma}^{\alpha} \operatorname{skew}\left(\widetilde{\boldsymbol{C}}^{e} \widetilde{\mathbf{Z}}^{\alpha}\right)
\end{aligned}
$$

Here $\widetilde{\boldsymbol{C}}^{e}$ is the elastic right Cauchy-Green tensor, $\widetilde{\mathbf{\Omega}}^{e}$ is the spin of the lattice, $\dot{\gamma}^{\alpha}$ is shear strain rate due to slip in $\alpha^{\text {th }}$ slip system, $\widetilde{\boldsymbol{Z}}^{\alpha}$ is the Schmid tensor of $\alpha^{\text {th }}$ slip system.

Evolution of plastic slip in terms of shear strain rate due to slip on each slip system, was defined using the power law: 


$$
\dot{\gamma}^{\alpha}=\dot{\gamma}_{0}^{\alpha}\left[\frac{\left|\tau^{\alpha}\right|}{\kappa_{s}^{\alpha}}\right]^{\frac{1}{m}} \operatorname{sign}\left(\boldsymbol{\tau}^{\alpha}\right)
$$

Here, $\dot{\gamma}_{0}^{\alpha}$ is the reference shear strain rate, $m$ is the strain rate dependence coefficient, $\boldsymbol{\tau}^{\alpha}$ is the resolved shear stress and $\kappa_{s}^{\alpha}$ is the current slip system strength, on $\alpha^{\text {th }}$ slip system.

Voce type hardening was incorporated in the model using the following evolution relation, which makes the slip system harden with the evolution of accumulated slip till a saturation value is reached, beyond which it will behave as a perfectly plastic material.

$$
\dot{\kappa}_{S}^{\alpha}=h_{0}\left(\frac{\kappa_{S, S}^{\alpha}-\kappa_{S}^{\alpha}}{\kappa_{s, S}^{\alpha}-\kappa_{S, 0}^{\alpha}}\right) \sum_{\alpha=1}^{N}\left|\dot{\gamma}^{\alpha}\right|, \kappa_{S, S}^{\alpha}=\kappa_{S, S 0}^{\alpha}\left[\frac{\sum_{\alpha}\left|\dot{\gamma}^{\alpha}\right|}{\dot{\gamma}_{s 0}}\right]^{1 \backslash m \prime}
$$

Where $\dot{\kappa}_{s}^{\alpha}$ is the current rate of hardening, $\kappa_{s}^{\alpha}$ is the current value of slip system strength, $h_{0}$ is the reference hardening coefficient, $\kappa_{S, S}^{\alpha}$ is the saturation value of strength which depends on the accumulated slip $\sum_{\alpha}\left|\dot{\gamma}^{\alpha}\right|$, and its evolution is given by a power law. The rest of the quantities, $\kappa_{s, 0}^{\alpha}, \kappa_{s, S 0}^{\alpha}, \dot{\gamma}_{s, 0}^{\alpha}$ and $m^{\prime}$ are the material parameters controlling the evolution of strength in the crystal. Critical resolved shear stress (CRSS) of each of the slip systems are assigned as $\kappa_{s}^{\alpha}(t=0)$ in (11).

\subsection{Parameter identification}

Material parameters for the two phases used in this study were found by calibrating the model against tensile test results of Ti-1023 alloy at room temperature and at a strain rate of 1 $\mathrm{mm} / \mathrm{min}$. As received alloy was heat treated at $700^{\circ} \mathrm{C}$ for 1 hour and then water quenched [22]. Volume fractions of the phases were found to be $40 \% \alpha_{p}$ and $60 \% \beta$ phase [23]. Since the alloy tested was polycrystalline, properties of 200 randomly oriented grains of $\alpha$ phase were assigned to one of the integration points and properties of the same number of grains of $\beta$ phase, having random Burger's orientation relation (BOR), with $\alpha$ grains were assigned to another integration point. A homogenised stress strain response was computed based on the volume fraction of the individual $40 \% \alpha_{p}$ and $60 \% \beta$ phase.

Figure 1 shows the comparison between experimental and the calibrated crystal plasticity model results. All material parameters used in RVE simulations are given in Table 1.

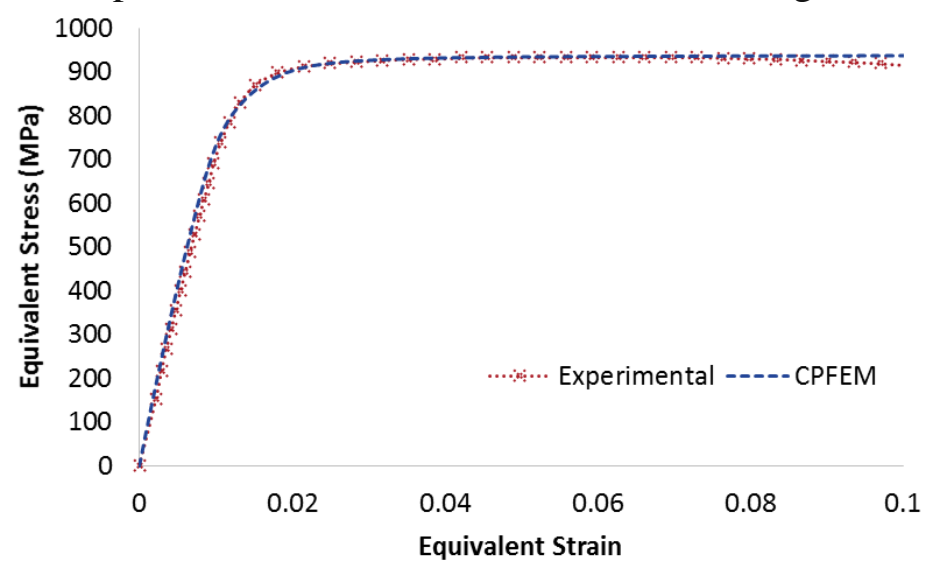

Figure 1 Model calibration with experimental results of Ti-1023 from the literature [22].

Table 1 Material parameters for CPFEM of Ti-1023 $\alpha-\beta$ phases

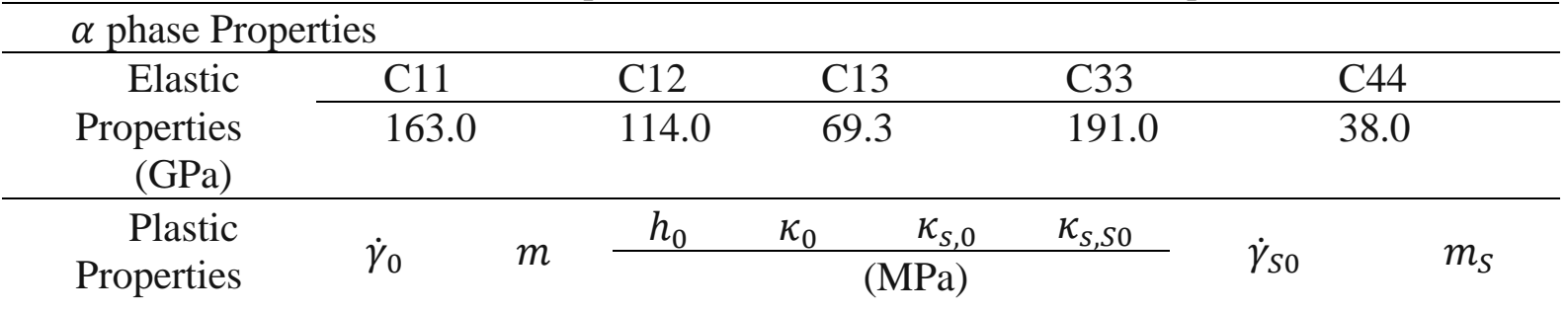




\begin{tabular}{|c|c|c|c|c|c|c|c|c|}
\hline Basal & 0.1 & 0.05 & 1 & 350 & 100 & 350 & $5 \times 1010$ & 0.005 \\
\hline Prismatic & 0.1 & 0.05 & 1 & 300 & 100 & 300 & $5 \times 1010$ & 0.005 \\
\hline Pyramidal & 0.1 & 0.05 & 1 & 750 & 100 & 750 & $5 \times 1010$ & 0.005 \\
\hline \multicolumn{9}{|c|}{$\beta$ phase Properties } \\
\hline \multirow{2}{*}{$\begin{array}{c}\text { Elastic } \\
\text { Properties }(\mathrm{GPa})\end{array}$} & C11 & \multicolumn{2}{|r|}{$\mathrm{C} 12$} & \multicolumn{2}{|l|}{$\mathrm{C} 44$} & & & \\
\hline & 140.0 & & 128.0 & 50.0 & & & & \\
\hline $\begin{array}{r}\text { Plastic } \\
\text { Properties }\end{array}$ & $\dot{\gamma}_{0}$ & $m$ & $h_{0}$ & $\kappa_{0}$ & $\frac{\kappa_{S, 0}}{\mathrm{P}_{2}}$ & $\kappa_{s, S 0}$ & $\dot{\gamma}_{S 0}$ & $m_{S}$ \\
\hline$\{110\}\langle 111\rangle$ & 0.1 & 0.05 & 1 & $\frac{(N)}{285^{(N}}$ & 100 & 285 & $5 \times 1010$ & 0.005 \\
\hline$\{110\}\langle 112\rangle$ & 0.1 & 0.05 & 1 & 320 & 100 & 320 & $5 \times 1010$ & 0.005 \\
\hline$\{110\}\langle 123\rangle$ & 0.1 & 0.05 & 1 & 380 & 100 & 380 & $5 \times 1010$ & 0.005 \\
\hline
\end{tabular}

\subsection{Geometry and Boundary Conditions}

A spherical void is modelled at the centre of a cube with side length given by:

$$
s=\left(\frac{\frac{4}{3} \pi r^{3}}{f_{0}}\right)^{\frac{1}{3}}
$$

Here, $s$ and $r$ are the side length and radius of the void and $f_{0}$ is the initial porosity. Two values of $f_{0}=0.001$ and 0.01 are used for the investigation. The cube with the void is then divided into two halves. One half was assigned $\alpha$ phase single crystal properties and the other half was assigned $\beta$ phase properties, given in Table 1 . The angle between the major loading direction, $F$ and normal to the phase interface, $N$ is termed as phase boundary inclination (PBI). Four PBIs were investigated from $90^{\circ}, \mathrm{PBI} 1$, to $0^{\circ}, \mathrm{PBI} 4$ with in an increment of $30^{\circ}$ at both values of $f_{0}$. BOR $(\overline{101})_{\beta} \|(0001)_{\alpha}$ and $[111]_{\beta} \|[2 \overline{1} \overline{1} 0]_{\alpha}$ was used for all PBIs and the Euler angles for the crystal orientation for each PBI are given in Table 2. BOR constrain the Basal plane of the HCP crystal to be parallel with the interface between phases, because of which crystal orientation of the $\alpha$ phase single crystal was changed as the PBI was rotated. Also, $\beta$ phase single crystal was updated accordingly as per BOR. Figure 2 shows geometries used in this study. Figure 2 (a) and (b) shows voids with two different initial porosities, and their location at the phase boundary of PBI 1 and PBI 2 respectively. Figure 2 (c-f) shows the geometries of PBI 1-4 respectively. Phases are shown in different shades, and the small HCP crystal was superimposed over the RVE to give an idea of the orientation of $\alpha$ phase single crystal.

All these geometries were tested at three levels of stress triaxiality values, $X=1 / 3,1$ and 3 . Stress triaxiality was kept constant using a multipoint constraint, MPC user subroutine of Abaqus software.

Table 2 Euler angles of $\alpha$ and $\beta$ phases for different phase boundary inclinations

\begin{tabular}{|c|c|c|c|c|c|c|c|}
\hline \multirow{2}{*}{ No. } & \multirow{2}{*}{ PBI } & \multirow[b]{2}{*}{$\Psi$} & $\alpha$ & \multirow[b]{2}{*}{$\phi$} & \multirow[b]{2}{*}{$\Psi$} & $\beta$ & \multirow[b]{2}{*}{$\phi$} \\
\hline & & & $\Theta$ & & & $\Theta$ & \\
\hline 1 & $90^{\circ}$ & $0^{\circ}$ & $0^{\circ}$ & $180^{\circ}$ & $324.74^{\circ}$ & $45.00^{\circ}$ & $180.00^{\circ}$ \\
\hline 2 & $60^{\circ}$ & $180^{\circ}$ & $30^{\circ}$ & $0^{\circ}$ & $289.73^{\circ}$ & $25.70^{\circ}$ & $138.27^{\circ}$ \\
\hline 3 & $30^{\circ}$ & $180^{\circ}$ & $60^{\circ}$ & $0^{\circ}$ & $231.59^{\circ}$ & $31.40^{\circ}$ & $073.67^{\circ}$ \\
\hline 4 & $0^{\circ}$ & $180^{\circ}$ & $90^{\circ}$ & $0^{\circ}$ & $210.00^{\circ}$ & $54.74^{\circ}$ & $045.00^{\circ}$ \\
\hline
\end{tabular}




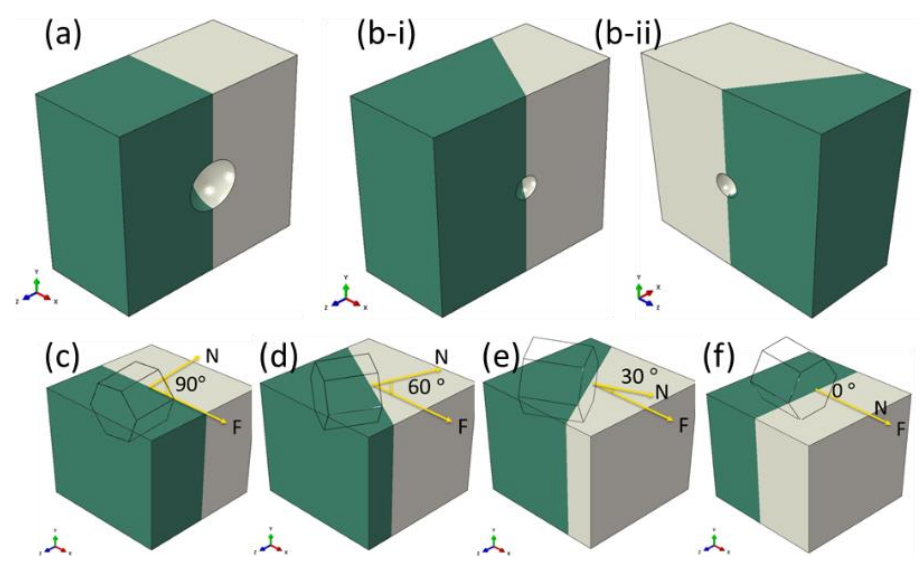

Figure 2 Some geometries used for RVE study, (a) PBI 1, $f_{0}=0.01$, (b-i, ii) Halves of PBI $2, f_{0}=0.001$, (c-f) PBI 1-4. Green colour represents $\alpha$ phase and beige colour is $\beta$ phase

\subsection{Results and Discussion}

\subsubsection{Effect of PBI on void growth}

Void Volume Fraction (VVF) evolution was found to be strongly dependent on the phase boundary inclination. Figure 3 (a-c) shows the effect of PBI, stress triaxiality and equivalent strain on the evolution of void volume fraction, with the contour plots for $f_{0}=0.01$ and $X=1 / 3$, 1 and 3 respectively. They helped in better quantifying the void growth with respect to the identified parameters and helped in formulating the model.

Figure 4 (a-d) shows the evolution of normalised VVF against applied equivalent strain at $f_{0}=0.001$ and 0.01 and stress triaxiality of $1 / 3,1$ and 3 in PBI 1, 2, 3 and 4 respectively. It can be seen that for all cases void growth increases exponentially with respect to applied equivalent strain. Rate of void growth increased when stress triaxiality was increased from $1 / 3$ to 3 . When the PBI was moved from 1-3, the void grows faster. For the case of PBI 4, evolution of VVF compared to other PBIs vary at different applied stress triaxiality. At $X=3$, void growth in PBI 4 is slowest as compared to other PBIs. But as the value of $X$ was decreased to 1 and then $1 / 3$, void growth in PBI 4 accelerated and becomes higher than PBI 2.

\subsubsection{Effect of initial porosity on void growth}

The effect of initial porosity on the evolution of void volume fraction in bicrystals of the $\alpha$ and $\beta$ phase is also shown in Figure 4 (a-d) for three levels of applied stress triaxiality 1/3, 1 and 3 in each of PBI 1, 2, 3 and 4 respectively. For higher initial porosity, slower void growth was observed for all PBIs and stress triaxialities with few exceptions. This trend is in agreement with the previous studies [14].

\section{PROPOSED MODEL}

Based on the findings made from the unit cell calculations, a model is sought which accounts for; applied strain $\left(\varepsilon_{e q}\right)$, stress triaxiality $(X)$, PBI $(\theta)$ and initial porosity. These parameters, except PBI have also been identified to influence the void growth and catered for in the model by Siddiq (2018). It was found from the RVE study that void growth increases exponentially with increasing equivalent strain. Rate of void growth was found to increase further as the stress triaxiality was increased and this increase is not linear with stress triaxiality, rather it increases exponentially with stress triaxiality. These trends are captured using a product of power laws of stress triaxiality and equivalent strains. Values of stress triaxiality were incremented by 1 to prevent reduction in void growth for $X<1$ for power higher than 1 . Also, equivalent strain was scaled before using in the power law to balance its contribution towards void growth with stress 
triaxiality. To insure the minimum value of normalised $\mathrm{VVF}$, at $\epsilon_{e q}=0$ remains 1 , it is added as a constant term. The resulting relation is given as:

$$
\frac{f}{f_{0}}=1+(1+X)^{A} \cdot\left(\frac{\varepsilon_{e q}}{C}\right)^{B}=\beta
$$

(a)

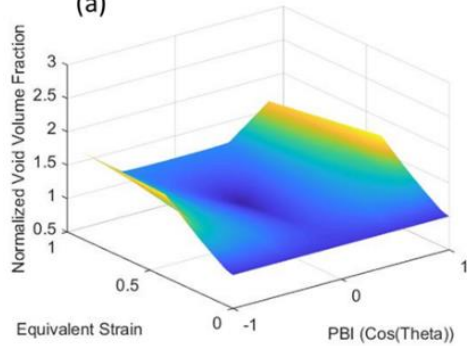

(b)

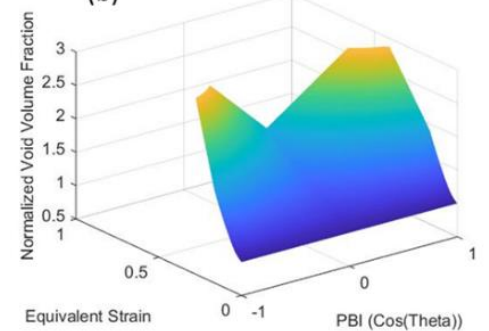

(c)

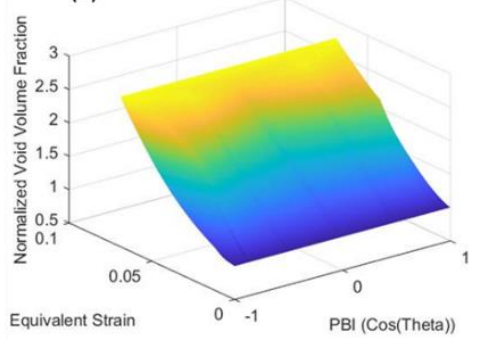

Figure 3: Caption?? I deleted I guess!
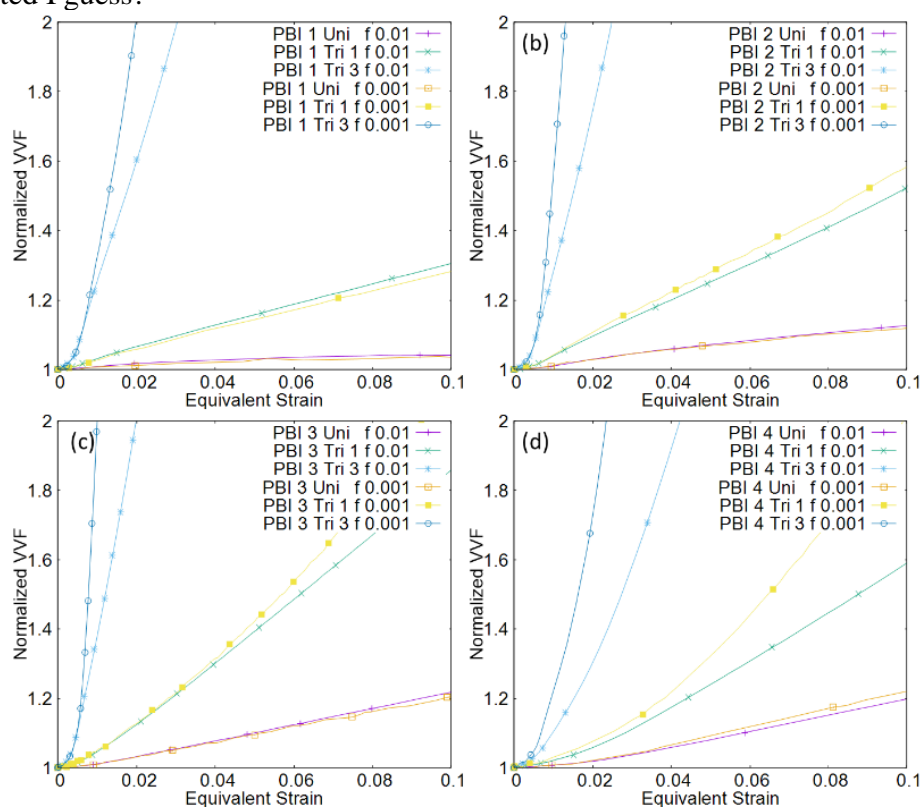

Figure 4 Effect of PBI on the normalized void volume fraction (VVF) evolution at $X=1 / 3,1$ and 3
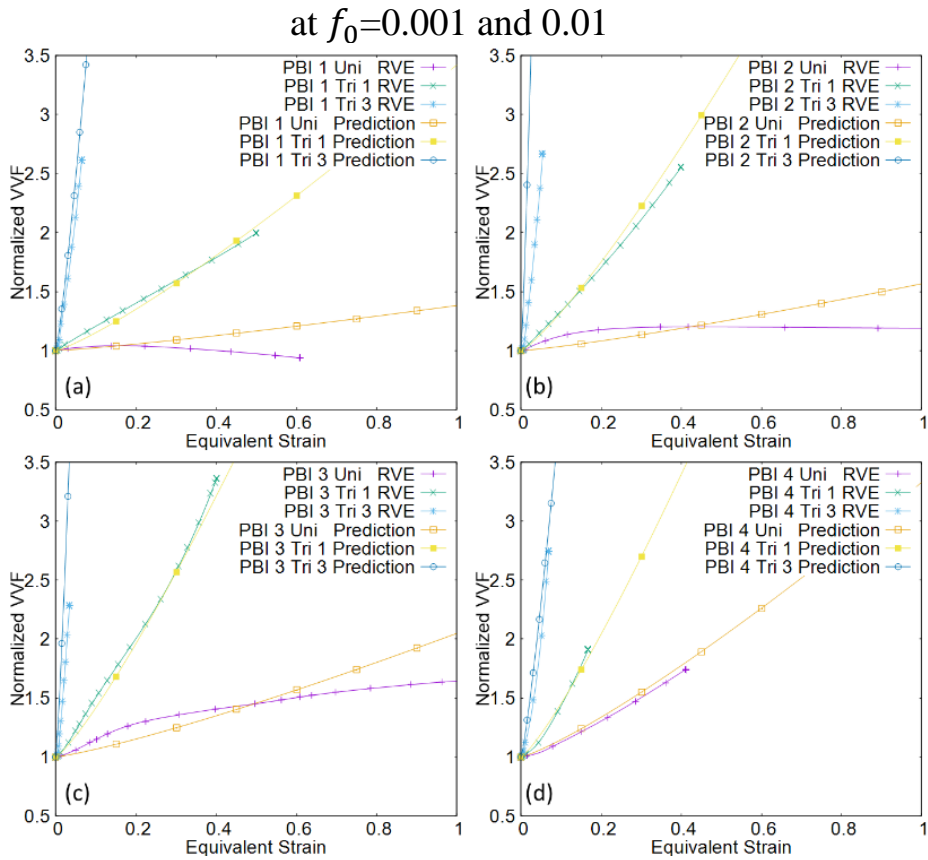

Figure 5 Comparison of normalized void volume fraction (VVF) evolution results of RVE simulations and proposed model for PBI 1, 2, 3 and 4 
Here $A, B$ and $C$ are parameters depending upon PBI, initial porosity and material properties. Values of $A, B$ and $C$ are first calibrated with unit cell results of $f_{0}=0.01$ and for each PBI separately to get the best fit with the proposed model. The value of $B$ was found to be equal for all PBIs but values of $A$ and $C$ vary for each PBI. A sech function was then used to relate the parameters $A$ and $C$ with the PBI getting following relations:

$$
\begin{aligned}
& A=D \operatorname{sech}(E \theta-F) \\
& C=G \operatorname{sech}(H \theta-I)
\end{aligned}
$$

Here coefficients $D, E, F, G, H$ and $I$ were calibrated with the values of $A$ and $C$ found for each PBI. $\beta$ in (13) is a non-dimensional strain like quantity which will be used to simulate material softening with void growth for future formulations. Parameters calibrated for $f_{0}=0.01$ are given in Table 3 for titanium alloy Ti-10V-2Fe-3Al. Figure 5 shows the comparison of unit cell results with the model prediction of normalized void volume fraction against applied equivalent strain for each PBI at three stress triaxialities $1 / 3,1$ and 3 at $f_{0}=0.01$. It can be seen that the proposed model is in good agreement with the unit cell results.

Table 3 Parameters of proposed model calibrated for $f_{0}=0.01$, Ti-1023 alloy

\begin{tabular}{lllllll}
\hline $\mathrm{B}$ & $\mathrm{D}$ & $\mathrm{E}$ & $\mathrm{F}$ & $\mathrm{G}$ & $\mathrm{H}$ & $\mathrm{I}$ \\
\hline 1.20 & 5.30 & 1.20 & 1.25 & 7.00 & 1.80 & 2.50 \\
\hline
\end{tabular}

\section{CONCLUSION}

Effects of initial porosity, stress triaxiality and PBI are studied in $\alpha-\beta$ titanium alloy (Ti$10 \mathrm{~V}-2 \mathrm{Fe}-3 \mathrm{Al}$ ) using a unit cell study, with the RVEs having a spherical void at the interface of $\alpha$ and $\beta$ phases. It was found that PBI has a strong effect on void growth along with initial porosity and stress triaxiality. Void growth increased when PBI was changed from $90^{\circ}$ to $0^{\circ}$. Void growth in $\beta$ phase was found to be higher than $\alpha$ phase in most PBIs. A model is formulated that accounts for all these effects, calibrated using the unit cell study results, good agreement is found between the two. The model gives a non-dimensional strain like function that can be used simulate softening due to void growth in dual phase titanium alloys.

\section{ACKNOWLEDGEMENT}

Author is thankful to University of Aberdeen for the award of Elphinstone Scholarship which covers the tuition fee of $\mathrm{PhD}$ study of author.

\section{DATA AVAILABILITY}

The raw/processed data required to reproduce these findings cannot be shared at this time as the data also forms part of an ongoing study.

\section{REFERENCES}

[1] G. Lütjering, J.C. Williams, Titanium : Engineering Materials and Processes, Springer, 2007. doi:10.1007/978-3-540-73036-1.

[2] M. Peters, J. Kumpfert, C.H. Ward, C. Leyens, Titanium alloys for aerospace applications, Adv. Eng. Mater. 5 (2003) 419-427. doi:10.1002/adem.200310095.

[3] R. Xie, N. Lin, P. Zhou, J. Zou, P. Han, Z. Wang, B. Tang, Surface damage mitigation of TC4 alloy via micro arc oxidation for oil and gas exploitation application: Characterizations of microstructure and evaluations on surface performance, Appl. Surf. Sci. 436 (2018) 467-476. doi:10.1016/J.APSUSC.2017.12.047.

[4] G.T. Terlinde, T.W. Duerig, J.C. Williams, Microstructure, tensile deformation, and fracture in aged ti 10V-2Fe-3Al, Metall. Trans. A. 14 (1983) 2101-2115. doi:10.1007/BF02662377. 
[5] S. Kanamori, M. Saito, E. Abe, T. Tagawa, T. Miyata, Effects of Triaxiality and Microstructure on Ductile Fracture of \&alpha;/\&beta; Titanium Alloys, J. Soc. Mater. Sci. Japan. 51 (2002) 900-905. doi:10.2472/jsms.51.900.

[6] A. Bhattacharjee, V.K. Varma, S. V Kamat, a K. Gogia, S. Bhargava, Influence of b Grain Size on Tensile Behavior and Ductile Fracture Toughness of Titanium Alloy Ti-10V-2Fe-3Al, Metall. Mater. Trans. A. 37 (2006) 1423. doi:10.1007/s11661-006-0087-x.

[7] D.F. Quinn, P.J. Connolly, M.A. Howe, P.E. McHugh, Simulation of void growth in WC-Co hardmetals using crystal plasticity theory, Int. J. Mech. Sci. 39 (1997) 173-183. doi:10.1016/0020-7403(96)000513.

[8] V.. Orsini, M.. Zikry, Void growth and interaction in crystalline materials, Int. J. Plast. 17 (2001) 13931417. doi:10.1016/S0749-6419(00)00091-7.

[9] G.P.P. Potirniche, J.L.L. Hearndon, M.F.F. Horstemeyer, X.W.W. Ling, Lattice orientation effects on void growth and coalescence in fcc single crystals, Int. J. Plast. 22 (2006) 921-942. doi:10.1016/j.ijplas.2005.06.003.

[10] W.H. Liu, X.M. Zhang, J.G. Tang, Y.X. Du, Simulation of void growth and coalescence behavior with 3D crystal plasticity theory, Comput. Mater. Sci. 40 (2007) 130-139. doi:10.1016/J.COMMATSCI.2006.11.009.

[11] S. Ha, K. Kim, Void growth and coalescence in f.c.c. single crystals, Int. J. Mech. Sci. 52 (2010) 863873. doi:10.1016/J.IJMECSCI.2010.03.001.

[12] S.K. Yerra, C. Tekog lu, F. Scheyvaerts, L. Delannay, P. Van Houtte, T. Pardoen, Void growth and coalescence in single crystals, Int. J. Solids Struct. 47 (2010) 1016-1029. doi:10.1016/J.IJSOLSTR.2009.12.019.

[13] M. Pushkareva, J. Adrien, E. Maire, J. Segurado, J. Llorca, A. Weck, Three-dimensional investigation of grain orientation effects on void growth in commercially pure titanium, Mater. Sci. Eng. A. 671 (2016) 221-232. doi:10.1016/J.MSEA.2016.06.053.

[14] U. Asim, M.A. Siddiq, M. Demiral, Void growth in high strength aluminium alloy single crystals: A CPFEM based study, Model. Simul. Mater. Sci. Eng. 25 (2017). doi:10.1088/1361-651X/aa5bcc.

[15] M.A. Siddiq, U. Bin Asim, A Crystal Plasticity Finite Element Method (CPFEM) based study to investigate the effect of microvoids in single crystalline aluminium alloy, in: Proc. 24th UK Conf. Assoc. Comput. Mech. Eng. 31 March - 01 April 2016, Cardiff Univ. Cardiff, 2016.

[16] H.-J. Guo, D.-F. Li, Crystal plasticity-based micromechanical finite element modelling of ductile void growth for an aluminium alloy under multiaxial loading conditions, Proc. Inst. Mech. Eng. Part L J. Mater. Des. Appl. (2018) 1464420718805828. doi:10.1177/1464420718805828.

[17] D.J. Savage, N. Chandola, O. Cazacu, B.A. McWilliams, M. Knezevic, Validation of recent analytical dilatational models for porous polycrystals using crystal plasticity finite element models with Schmid and non-Schmid activation laws, Mech. Mater. 126 (2018) 148-162. doi:10.1016/J.MECHMAT.2018.08.004.

[18] Z. Li, W. Guo, The influence of plasticity mismatch on the growth and coalescence of spheroidal voids on the bimaterial interface, Int. J. Plast. 18 (2002) 249-279. doi:10.1016/S0749-6419(00)00078-4.

[19] M. Yang, X. Dong, Simulation of lattice orientation effects on void growth and coalescence by crystal plasticity, Acta Metall. Sin. (English Lett. 22 (2009) 40-50. doi:10.1016/S1006-7191(08)60069-1.

[20] W. Jeong, C.-H. Lee, J. Moon, D. Jang, M.-G. Lee, Grain Scale Representative Volume Element Simulation to Investigate the Effect of Crystal Orientation on Void Growth in Single and Multi-Crystals, Metals (Basel). 8 (2018). doi:10.3390/met8060436.

[21] E.B. Marin, On the Formulation of a Crystal Plasticity Model, Sandia National Laboratories, Livermore, 2006.

[22] W. Chen, Z. Song, Q. Sun, L. Xiao, W. She, J. Sun, P. Ge, Effect of Solution Treatment Temperature on Trigger Stress for Stress Induced Martensitic Transformation in Ti-10V-2Fe-3Al Alloy, J. Solid Mech. Mater. Eng. 4 (2010) 1296-1305. doi:10.1299/jmmp.4.1296.

[23] W. Shihong, S. Guiqin, Ti-10V-2Fe-3Al Alloy Solid Solution Ageing, Structure and Properties, Rare Met. Mater. Eng. (1990) 26-34.

[24] A. Siddiq, A porous crystal plasticity constitutive model for ductile deformation and failure in porous single crystals, Int. J. Damage Mech. 0 (2018) 1056789518757294. doi:10.1177/1056789518757294. 